\title{
Erratum to: Fuelling the English breakfast: hidden energy flows in the Anglo-Danish trade 1870-1913
}

\author{
Sofia Teives Henriques ${ }^{1}$ Paul Warde ${ }^{2}$
}

Published online: 27 June 2017

(C) Springer-Verlag GmbH Germany 2017

Erratum to: Reg Environ Change

DOI 10.1007/s10113-017-1166-9

The published online version contains mistake. An entry is missing from Table 6. Value 2.7 should be in row 2.1 (Feed (metabolizable)) under column (Eggs PJ), as shown below.

The online version of the original article can be found at http://dx.doi.org/ 10.1007/s10113-017-1166-9

Sofia Teives Henriques

sofiathenriques@gmail.com

Paul Warde

psw1000@cam.ac.uk

1 Department of Economic History, Lund University, Lund, Sweden

2 University of Cambridge, Cambridge, UK 
Table 6 Decomposition of energy embodied in Danish Exports to UK of butter, pork and bacon and eggs, 1913, petajoules (PJ)

\begin{tabular}{|c|c|c|c|c|c|c|}
\hline & Origin & Place of use & $\begin{array}{l}\text { Butter } \\
\text { PJ }\end{array}$ & $\begin{array}{l}\text { Pork and bacon } \\
\text { PJ }\end{array}$ & $\begin{array}{l}\text { Eggs } \\
\text { PJ }\end{array}$ & $\begin{array}{l}\text { Total } \\
\text { PJ }\end{array}$ \\
\hline \multicolumn{7}{|l|}{ 1. Coal to industrial processing } \\
\hline Mining the coal & UK & UK & 0.2 & 0.0 & 0.0 & 0.2 \\
\hline Cream separation & UK & DK & 2.6 & 0.0 & 0.0 & 2.6 \\
\hline Slaughterhouses & UK & DK & 0.0 & 0.8 & 0.0 & 0.8 \\
\hline \multicolumn{7}{|l|}{ 2. Domestic feed } \\
\hline 2.1. Feed (metabolizable) & DK & DK & 20.0 & 10.1 & 2.7 & 32.8 \\
\hline \multicolumn{7}{|l|}{ 2.2. Hidden energy in feed } \\
\hline Animal labour & DK & DK & 1.8 & 2.8 & 1.2 & 5.9 \\
\hline Fossil fuels (field, oil cakes) & UK & DK & 0.3 & 0.1 & 0.0 & 0.5 \\
\hline Mining the coal & UK & UK & 0.0 & 0.0 & 0.0 & 0.0 \\
\hline \multicolumn{7}{|l|}{ Domestic fertilizers } \\
\hline a. Coal to fertilizers & UK & DK & 0.1 & 0.1 & 0.0 & 0.2 \\
\hline b. Mining the coal & UK & UK & 0.0 & 0.0 & 0.0 & 0.0 \\
\hline Foreign fertilizers & $\mathrm{NO}, \mathrm{CL}$ & & & & & \\
\hline a. Coal, hydro-power & CL, UK, NO & & 0.2 & 0.3 & 0.1 & 0.6 \\
\hline \multicolumn{7}{|l|}{ 3. Foreign feed } \\
\hline 3.1. Feed (metabolizable) & USA, RU, DE & DK & 5.0 & 2.7 & 1.3 & 9.0 \\
\hline \multicolumn{7}{|l|}{ 3.2. Hidden energy in feed } \\
\hline Animal labour & USA, RU, DE & USA, RU, DE & 0.2 & 1.2 & 0.6 & 2.0 \\
\hline Fossil fuels & unknown & USA, RU, DE & 2.7 & 0.2 & 0.0 & 2.8 \\
\hline Energy to fertilizers & unknown & USA, RU, DE & 0.0 & 0.1 & 0.1 & 0.2 \\
\hline Total & & & 33.1 & 18.4 & 6.1 & 57.6 \\
\hline \multicolumn{7}{|l|}{ By origin } \\
\hline Denmark & & & 21.9 & 12.9 & 3.9 & 38.7 \\
\hline UK & & & 3.2 & 1.0 & 0.1 & 4.3 \\
\hline Others & & & 8.1 & 4.4 & 2.1 & 14.6 \\
\hline$\%$ embodied energy from Denmark & & & $66 \%$ & $70 \%$ & $64 \%$ & $67 \%$ \\
\hline$\%$ embodied energy used in Denmark & & & $90 \%$ & $91 \%$ & $87 \%$ & $90 \%$ \\
\hline
\end{tabular}

Sources: own construction from sources listed in text. Trade partners taken from SD (1968); 1969)

DK Denmark, RU Russia, DE Germany, NO Norway, CL Chile 